\title{
Implementation of generalized quantum measurements: superadditive quantum coding, accessible information extraction, and classical capacity limit
}

\author{
Masahiro Takeoka, ${ }^{1,2}$ Mikio Fujiwara, ${ }^{1,2}$ Jun Mizuno, ${ }^{1,2}$ and Masahide Sasaki ${ }^{1,2}$ \\ ${ }^{1}$ Communications Research Laboratory, Koganei, Tokyo 184-8795, Japan \\ ${ }^{2}$ CREST, Japan Science and Technology Corporation, Shibuya, Tokyo 150-0002, Japan
}

(Dated: August 14, 2018)

\begin{abstract}
Quantum information theory predicts that when the transmission resource is doubled in quantum channels, the amount of information transmitted can be increased more than twice by quantum channel coding technique, whereas the increase is at most twice in classical information theory. This remarkable feature, the superadditive quantum coding gain, can be implemented by appropriate choices of code words and corresponding quantum decoding which requires a collective quantum measurement. Recently, the first experimental demonstration was reported [Phys. Rev. Lett. 90, 167906 (2003)]. The purpose of this paper is to describe our experiment in detail. Particularly, a design strategy of quantum collective decoding in physical quantum circuits is emphasized. We also address the practical implication of the gain on communication performance by introducing the quantum-classical hybrid coding scheme. We show how the superadditive quantum coding gain, even in a small code length, can boost the communication performance of conventional coding technique.
\end{abstract}

PACS numbers: 03.67.-a, 03.65.Ta, 89.70.+c

\section{INTRODUCTION}

It is a fundamental problem in information science what is the most efficient way of transmitting information with a minimum of transmission resources. The amount of information transmissible through a communications channel is determined by the noise characteristics of the channel and by the quantities of available transmission resources. In classical communication theory 1, 2, 3], the amount of transmissible information can be increased twice at most when the transmission resource (e.g. the code length, the signal power, the bandwidth) is doubled, for the fixed noise characteristics of the channel. In quantum communication theory, however, this is not true in general, that is, the amount of information transmitted can be increased even more than twice. This feature is called the superadditivity of the capacity of quantum channel $4,5,6,7,8,9]$.

The superadditivity becomes essential in any transmission of signals at the quantum level where ambiguity among signals is a matter of non-commutativity of the density matrices, i.e. $\hat{\rho}_{0} \hat{\rho}_{1} \neq \hat{\rho}_{1} \hat{\rho}_{0}$, rather than any classical noises such as thermal noise. One typical example is deep space optical communications. Inter-satellite optical link is expected to achieve a high transmission rate that cannot be achieved by the radio- or micro-wave links, e.g. to realize data transmission from a space-telescope with billion pixels or real time communications over the planets. In a deep space optical link, the sender prepares coherent state signals with as large amplitude as possible allowed by a limited power supply. Such signals are, however, extremely weakened at the receiving end, typically less than a few photons per pulse, due to the beam divergence and energy loss. Since the energy quantum of carriers is greater than that of thermal noise in optical domain, i.e. $\hbar \omega>k_{\mathrm{B}} T$, physical states of carriers can be described by pure quantum states in good ap- proximation. For example, the binary phase shift keyed signals to convey classical letters 0 and 1 , respectively, are represented by the coherent states $\hat{\rho}_{0}=|\alpha\rangle\langle\alpha|$ and $\hat{\rho}_{1}=|-\alpha\rangle\langle-\alpha|$, respectively. For weak coherent pulses, the state overlap $\langle\alpha \mid-\alpha\rangle$ becomes non-negligible, i.e. $\hat{\rho}_{0}$ and $\hat{\rho}_{1}$ are noncommuting. According to the uncertainty principle, noncommuting density matrices can never be distinguished perfectly. This imposes an inevitable error in signal detection even in an ideal communications system 10]. Actually, when $|\alpha|^{2} \leq 3, \hat{\rho}_{0}$ and $\hat{\rho}_{1}$ can not be distinguished at a bit error rate less than $10^{-6}$, which is a typical error free criterion in deep space communications.

Historically, an extension of communication theory into quantum domain including this aspect of ambiguity has been explored since 1960's 11, 12, 13, 14]. In 1973, Holevo derived the quantity that bounds the upper limit of the capacity of a quantum communications channel [14]. It was recently shown that this so-called Holevo bound is an achievable rate, that is, the exact expression of the capacity 15, 16, 17]. Classical communication theory 1, 2, 3] describes the special case where the signals are given by commuting density matrices. The distinctive characteristics of quantum theory of capacity is the great emphasis on quantum decoding process to extract information from block sequences of noncommuting density matrices. The essence of the optimal decoding is the use of a process of entangling letter states constituting code words prior to measurement to enhance the distinguishability of signals. Such a process is a quantum computation on code word states. This socalled quantum collective decoding is a new aspect, not found in conventional coding techniques, and leads to a larger capacity. This is called the superadditive quantum coding gain (SQCG) [4, 5, 6, 7, 8, 9] in a quantum channel since the length $n$ quantum coding makes capacity more than $n$ times larger from the capacity achievable only by conventional coding. Taking it into account, the 
capacity is defined as the maximum rate of the mutual information for a (quantum) code of length $n$ divided by the length $n$ in the limit of $n \rightarrow \infty$ for asymptotic error free transmission.

The theory of capacity, however, generally gives no guidance on how to construct codes that approach the capacity. A practical problem is then to find good codes to attain a large SQCG in a small block length. This must be an important issue in any communications and information-processing systems when they work at the quantum level, which is expected in a few decades considering recent exponential growth of info-communication demands. However, little attention has been paid to this topic so far. Only several coding schemes have been proposed to exhibit SQCG [5, 6, 7, 8, 9] and the first experimental demonstration has recently been reported by the authors [18. The purpose of the present paper is to give detailed information that was abbreviated or omitted in our previous letter. An attention is particularly paid to describe the strategy how to implement the quantum measurements used in our experiments by logical and physical quantum circuits. We also describe the detailed discussion of the implications of SQCG in small code length on practical communication performances.

The paper is organized as follows. In Sec. III we remind readers of several capacities of quantum channels studied to date, and explain our scenario. In Sec. III the basic notion for capacity theorem and SQCG are briefly explained. In Sec. IV] we discuss how we designed logical and physical quantum circuits for our SQCG experiment, which was omitted in our previous letter. Section $\nabla$ describes in detail our experiment of SQCG reported in Ref. [18]. We also show the experimental results about the separable quantum measurements attaining the single-shot capacity and the accessible information for comparison. In Sec. VI we discuss how SQCG, even the small gain demonstrated in length two coding, can boost a communication performance attained by conventional coding technique. The idea is based on quantumclassical hybrid coding (QCHC) which was briefly mentioned in our previous letter [18]. Theoretical details on the methodology of QCHC are given. Section VII is for concluding remarks.

\section{CAPACITIES FOR QUANTUM CHANNELS}

Since Shannon's capacity theory was extended into generic quantum states in Refs. [15, 16, 17], the capacity theory is further extended to include new auxiliary resources of entangled particles, new quantum protocols, and a new object to be transmitted, i.e. intact quantum state [19]. The notion of the capacity for quantum channels is now classified into two categories; 1) the classical capacity for transmitting conventional (classical) alphabet, and 2) the quantum capacity for transmitting quantum alphabet (unknown quantum states). For both categories entanglement assisted protocols may be considered, namely superdense coding [20] and quantum teleportation [21], respectively. Our concern is the first category, i.e. the classical capacity.

Depending on whether additional entanglement resources are brought into play or not, the classical capacity is classified into two kinds, namely, the entanglementassisted capacity $C_{\mathrm{E}}$ and the ordinary capacity $C$. The former is defined for a quantum channel with the help of unlimited prior entanglement sharing between the sender and the receiver 22]. The latter is defined for a quantum channel with the help of any allowed quantum operations at the sender (quantum encoding) and the receiver (quantum decoding), but without any prior source sharing. Both schemes assume multiple uses of the channel, that is, coding, and the capacity is defined as the maximum amount of transmissible information per channel use. It should be noted that shared entanglement is not regarded as the transmission resources in the definition of the entanglement-assisted capacity $C_{\mathrm{E}}$. This type of classification can simplify the study of several distinct capacities and their relation including the quantum capacities [22].

From a practical point of view, on the other hand, it is not realistic to expect the assistance of unlimited external resources. To predict the highest transmission rate in realistic situations, all the physical entities used for transmission, such as the shared entanglement, must be included in the elements constituting a communications channel. In this situation, the ordinary capacity $C$ is appropriate to evaluate the communication performance of the channel since it imposes the power constraint condition of the total physical resources.

Keeping such backgrounds in mind, we restrict our discussion to the following protocol. The sender transmits classical alphabet in classically encoded format, i.e. in separable tensor product states (code word states) made of a given set of letter states, and these code word states are still separable at the receiving end. No prior entanglement is shared between the sender and the receiver. The receiver may apply any quantum operations to the received code word states. In fact, it is known that effective quantum coding is that the receiver entangles the letter states prior to detection. This is called quantum decoding and contributes to SQCG which is never observed in any classical coding. Such scenario is within the framework of the ordinary capacity $C$, and exactly the case that the capacity theories of Refs. 15, 16, 17] concern. The reasons for choosing such protocol and for excluding quantum encoding and prior entanglement sharing are 1) concrete quantum coding schemes are known only for such protocol at present, and 2) it fits better to practical motivations introduced in Sec. [1 since it does not necessarily require a transmission of nonclassical state signals. The main task in this paper is, therefore, the demonstration of the quantum decoding process. 


\section{SUPERADDITIVE CODING GAIN}

A practical mean for effective communications is coding, that is, representing alphabet by sequences of simple letters such as $\{0,1\}$. Alphabets to be transmitted are represented by code words which are sequences of a given set of letters $\left\{x_{0}, \ldots, x_{L-1}\right\}$ such as the binary set $\{0,1\}$. The transmitter modulates a signal carrier into one of $L$ states $\left\{\hat{\rho}_{0}, \ldots, \hat{\rho}_{L-1}\right\}$ according to the input letter. If the letter states $\left\{\hat{\rho}_{0}, \ldots, \hat{\rho}_{L-1}\right\}$ appear as orthogonal states at the receiving end, then they can be distinguished perfectly and $\log _{2} L$ bits of information, which is the maximum Shannon entropy of the set $\left\{x_{0}, \ldots, x_{L-1}\right\}$, can be faithfully retrieved per letter. This is, however, not the case in general. A channel is usually subject to various types of noise disturbances. In order to transmit information reliably through a channel with finite errors, one must introduce some redundancy in code word representation prior to transmission so as to allow the correction of errors at the receiving side. This entails adding some redundant letters to the code words and hence increases their length. This is channel coding.

First, the source encoder converts the original message into a sequence of the letters in the given set $\left\{x_{0}, \ldots, x_{L-1}\right\}$, and then the channel encoder divides it into blocks of length $k$ (message blocks). Each block is supplemented by an additional block (redundant block) of $n-k(n>k)$ letters to compose a channel code word $\left\{\mathbf{x}_{i}\right\}$ :

$$
\begin{aligned}
\mathbf{x}_{i}= & \overbrace{x_{1}^{(i)} x_{2}^{(i)} \cdots x_{k}^{(i)}}^{\text {message block redundant block }} \overbrace{x_{k+1}^{(i)} x_{k+2}^{(i)} \cdots x_{n}^{(i)}} \\
& \left(\text { for } i=1,2, \ldots, L^{k}\right) .
\end{aligned}
$$

Note that although there are $L^{n}$ possible sequences of length $n$ in total, only part of them, i.e. $L^{k}$ sequences, are used as code words. This redundancy, together with appropriate encoding and decoding, allows us to recover possible errors in transmission. The amount of information conveyed by the above code words is $K=k \log _{2} L$ bits. The transmission rate is then defined by $R=$ $K / n=(k / n) \log _{2} L$ bits/letter. For a channel with a capacity $C$ bits/letter, it is possible [1, 2, 3] within the rate $R=K / n<C$ to reproduce the $K$ bits of messages with an error probability as small as desired by appropriate encoding and decoding in the limit $n \rightarrow \infty$.

A mathematical model of a channel is specified by a set of possible outputs $\{y\}$ from the channel and a channel matrix in which each matrix element is given by the conditional probability $P(y \mid x)$ of having $y$ given the input $x$. Each input letter $x$ is used with a priori probability $P(x)$. The probability of having $y$ is then given by

$$
P(y) \equiv \sum_{x} P(y \mid x) P(x)
$$

To define the capacity, Shannon introduced the mutual information [1]. This is defined between the in- put variable $X=\{x ; P(x)\}$ and the output variable $Y=\{y ; P(y)\}$ as

$$
I(X: Y)=\sum_{x} P(x) \sum_{y} P(y \mid x) \log \left[\frac{P(y \mid x)}{\sum_{x^{\prime}} P\left(x^{\prime}\right) P\left(y \mid x^{\prime}\right)}\right] .
$$

In classical information theory, one considers coding for a given and fixed channel model $\{P(y \mid x)\}$. The decoding error of code words $\left\{\mathbf{x}_{1}, \ldots, \mathbf{x}_{L^{k}}\right\}$ can be calculated based on the probability distributions $\{P(x)\}$ and $\{P(y \mid x)\}$. The capacity (for a memoryless channel) is defined as the maximum mutual information with respect to the prior distribution of the letters $P(x)$,

$$
C=\max _{\{P(x)\}} I(X: Y)
$$

In the quantum context, however, only the input variable $X$ and the corresponding set of quantum states at the receiver's hand denoted as $\left\{\hat{\rho}_{x}\right\}$ are given. The output variable $Y$ is to be sought for the best quantum measurement. A quantum measurement process can mathematically be described by a set of non-negative Hermitian operators $\left\{\hat{\Pi}_{y}\right\}$ satisfying the probability conservation relation $\sum_{y} \hat{\Pi}_{y}=\hat{I}$, so-called the positive operator valued measure (POVM). The channel matrix is then given by

$$
P(y \mid x) \equiv \operatorname{Tr}\left(\hat{\Pi}_{y} \hat{\rho}_{x}\right)
$$

and now one can define the maximum extractable information

$$
I_{\mathrm{Acc}}=\max _{\left\{\hat{\Pi}_{y}\right\}} I(X: Y),
$$

which is called the accessible information. More generally, the quantity further maximized with respect to the prior probability

$$
C_{1}=\max _{\{P(x)\}} \max _{\left\{\hat{\Pi}_{y}\right\}} I(X: Y)
$$

specifies the classical limit of the capacity when the given initial channel $\{P(y \mid x)\}$ is used with classical channel coding [23]. It is this quantity that limits the performance of all modern communications systems. This is, however, not the ultimate capacity allowed by quantum mechanics.

The code words $\{\mathbf{x}\}$ are now conveyed by the quantum states in a tensor product of the letter states, $\hat{\Psi}_{\mathbf{x}}=\hat{\rho}_{x_{1}} \otimes$ $\cdots \otimes \hat{\rho}_{x_{n}}$. To decode them, one may design the best quantum measurement allowed by quantum mechanics. This is described by the POVM $\left\{\hat{\Pi}_{\mathbf{y}}\right\}$ on the extended space where $\{\mathbf{y}\}$ are decoded code words. The channel matrix for this extended channel is given by

$$
P(\mathbf{y} \mid \mathbf{x}) \equiv \operatorname{Tr}\left(\hat{\Pi}_{\mathbf{y}} \hat{\Psi}_{\mathbf{x}}\right)
$$


One may then define the mutual information for this extended channel by

$I\left(X^{n}: Y^{n}\right)=\sum_{\mathbf{x}} P(\mathbf{x}) \sum_{\mathbf{y}} P(\mathbf{y} \mid \mathbf{x}) \log \left[\frac{P(\mathbf{y} \mid \mathbf{x})}{\sum_{\mathbf{x}^{\prime}} P\left(\mathbf{x}^{\prime}\right) P\left(\mathbf{y} \mid \mathbf{x}^{\prime}\right)}\right]$.

Further one can define the quantity

$$
C_{n}=\max _{\{P(\mathbf{x})\}} \max _{\left\{\hat{\Pi}_{\mathbf{y}}\right\}} I\left(X^{n}: Y^{n}\right),
$$

which we refer to the capacity of order $n$. The superadditivity of quantum channel is then expressed as

$$
C_{n}>n C_{1}
$$

The capacity of quantum channel as the maximum rate of error free transmission is defined by

$$
C=\lim _{n \rightarrow \infty} \frac{C_{n}}{n}
$$

The property of Eq. (11) was first predicted by Holevo based on the random coding technique [4]. Peres and Wootters conjectured that

$$
I\left(X^{2}: Y^{2}\right)>2 \max _{\left\{\hat{\Pi}_{y}\right\}} I(X: Y),
$$

by using the ternary symmetric states of qubit [5]. In these works, the importance of using quantum collective measurement on a block sequence of code word state was emphasized. The first rigorous example of the superadditivity was given by Sasaki et al. for the binary pure letter states [6], where the quantum channel showing

$$
I\left(X^{3}: Y^{3}\right)>3 C_{1}
$$

was explicitly demonstrated. Since then several examples of quantum code construction with the superadditivity were clarified [6, 7, 8, 9].

The important observation of the superadditivity is that the property

$$
P(\mathbf{y} \mid \mathbf{x}) \neq P\left(y_{1} \mid x_{1}\right) \cdots P\left(y_{n} \mid x_{n}\right)
$$

generally holds when an appropriate collective POVM is chosen. This is a kind of memory effect of the extended channel. When the measurement is made by projection onto separable bases, such a memory effect never takes place. Projection onto appropriate entangled bases induces quantum interferences among the code word states to reduce the ambiguity among the signals. The memory effect is a direct consequence of this quantum interference of block codes, that is, exactly the effect of the entanglement. Realization of such a quantum collective decoding generally requires quantum computation to entangle the letter states [24].

\section{MODEL FOR PROOF-OF-PRINCIPLE DEMONSTRATION: QUBIT TRINE}

Quantum collective decoding on quantum particles, that is, entangling quantum particles, is something very hard to realize at present even for two particles. In addition, the gains predicted for short length codes are very small. Here we consider how one can demonstrate the principle of quantum collective decoding. We deal with the noiseless channel model in which only the noncommutativity of the signals causes the transmission error.

The simplest set of letters is the binary set of pure states $\left\{\left|\psi_{0}\right\rangle,\left|\psi_{1}\right\rangle\right\}$ where the overlap between the letters is $\left\langle\psi_{0} \mid \psi_{1}\right\rangle=\kappa$. Only for this set, the classical capacity limit $C_{1}$ is known with rigorous mathematical proof 25, 26, 27]. The very first step is the length two coding. We have four possible tensor product sequences $\left\{\left|\psi_{0}\right\rangle\left|\psi_{0}\right\rangle,\left|\psi_{0}\right\rangle\left|\psi_{1}\right\rangle,\left|\psi_{1}\right\rangle\left|\psi_{0}\right\rangle,\left|\psi_{1}\right\rangle\left|\psi_{1}\right\rangle\right\}$. Buck et al. [8] showed that, by choosing three of them as code word states, the channel exhibits the superadditivity depending on the overlap $\kappa$. Unfortunately, the predicted maximum SQCG was only $I_{2} / 2-C_{1}=5.2 \times 10^{-4}$ bits and it seems too small to be observed experimentally. In the length three coding [6], the four code words were picked up from eight possible sequences so that the Hamming distance between each code word is equal $(d=2)$ to show the gain described in Eq. (14). In this case, the maximum gain was predicted to be $9 \times 10^{-3}$ bits. Although the gain is bigger than that in the length two coding, entangling three qubits for the quantum collective decoding requires more than ten steps of quantum gating, which seems to be difficult to realize.

Therefore, we consider the second simplest case, the qubit trine signals. The qubit trine consists of the ternary symmetric letter states of a qubit $\left\{\left|\psi_{0}\right\rangle,\left|\psi_{1}\right\rangle,\left|\psi_{2}\right\rangle\right\}$. It is this model which we use to demonstrate our experimental steps toward SQCG in the next section. It should be noted that the dimensionality (2-dim. ) is essential here. If the ternary states are defined in a higher dimensional space than three, such as the lifted trine, we do not know the exact value of $C_{1}$. In addition, according to our numerical studies, SQCG in terms of the mutual information appears smaller compared to that in the qubit trine case. The qubit trine is defined by the letter state set $\left\{\left|\psi_{0}\right\rangle,\left|\psi_{1}\right\rangle,\left|\psi_{2}\right\rangle\right\}$ with

$$
\begin{aligned}
\left|\psi_{0}\right\rangle & =|0\rangle, \\
\left|\psi_{1}\right\rangle & =-\frac{1}{2}|0\rangle-\frac{\sqrt{3}}{2}|1\rangle, \\
\left|\psi_{2}\right\rangle & =-\frac{1}{2}|0\rangle+\frac{\sqrt{3}}{2}|1\rangle,
\end{aligned}
$$

where $\{|0\rangle,|1\rangle\}$ is the orthonormal basis set. They are represented in Fig. 1(a).

The accessible information, defined by Eq. (6), for this set with equal prior probabilities is found to be $I_{\text {Acc }}=0.5850$ bits with a rigorous proof $[28]$. The optimal measurement strategy is described by the nonorthog- 


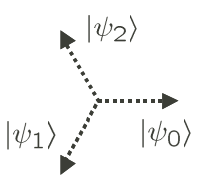

(a)

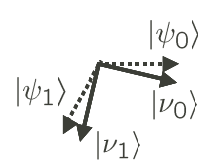

(b)

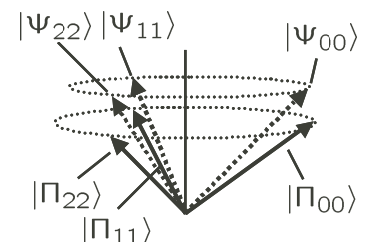

(c)
FIG. 1: Geometrical representation of several sets of quantum state vectors and measurement vectors. (a) The ternary symmetric letter states (qubit trine). (b) The letter (dotted arrows) and the measurement (solid arrows) state vectors to attain the $C_{1}$. (c) The code word (dotted arrows) and decoding (solid arrows) state vectors represented in a real three dimensional space.

onal basis set $\left\{\left|\omega_{0}\right\rangle,\left|\omega_{1}\right\rangle,\left|\omega_{2}\right\rangle\right\}$ of

$$
\begin{aligned}
& \left|\omega_{0}\right\rangle=-\sin \frac{\gamma_{\mathrm{Acc}}}{2}|1\rangle, \\
& \left|\omega_{1}\right\rangle=-\frac{1}{\sqrt{2}}|0\rangle+\frac{1}{\sqrt{2}} \cos \frac{\gamma_{\mathrm{Acc}}}{2}|1\rangle, \\
& \left|\omega_{2}\right\rangle=\frac{1}{\sqrt{2}}|0\rangle+\frac{1}{\sqrt{2}} \cos \frac{\gamma_{\mathrm{Acc}}}{2}|1\rangle,
\end{aligned}
$$

where $\gamma_{\text {Acc }}$ is defined by

$$
\cos \frac{\gamma_{\mathrm{Acc}}}{2}=\cot \frac{\pi}{3}
$$

This is a typical example of generalized quantum measurement, and was demonstrated in laboratory for polarization qubit of a photon in Refs. [29, 30]. The functional meaning of this quantity $I_{\mathrm{Acc}}$ is as follows. If the receiver applies this detection separately on each letter states (separable decoding), and encoding is made such that each letter states occurs with equal probabilities in the set of code words, then the maximum transmission rate for error free transmission is $I_{\mathrm{Acc}}=0.5850$ bits/letter.

One may further optimize the quantity besides the detection strategy. For the ternary set, $C_{1}$ has been carefully studied and evaluated to be 0.6454 bits [27, 31]. This is attained by discarding one of the three letters and using only two of them, say $\left\{\left|\psi_{0}\right\rangle,\left|\psi_{1}\right\rangle\right\}$, with equal probability $1 / 2$ and applying the measurement described by the orthonormal basis,

$$
\begin{aligned}
& \left|\nu_{0}\right\rangle=\frac{\sqrt{2+\sqrt{3}}}{\sqrt{3}}\left|\psi_{0}\right\rangle+\frac{\sqrt{2-\sqrt{3}}}{\sqrt{3}}\left|\psi_{1}\right\rangle, \\
& \left|\nu_{1}\right\rangle=\frac{\sqrt{2-\sqrt{3}}}{\sqrt{3}}\left|\psi_{0}\right\rangle+\frac{\sqrt{2+\sqrt{3}}}{\sqrt{3}}\left|\psi_{1}\right\rangle .
\end{aligned}
$$

This is schematically illustrated in Fig. 1(b).

Now we construct the length two coding. For the qubit trine letters, there are nine possible sequences. Peres and Wootters showed [5] that if one uses only three of them, which are

$$
\begin{aligned}
\left|\Psi_{x x}\right\rangle= & \left|\psi_{x}\right\rangle \otimes\left|\psi_{x}\right\rangle \\
= & \frac{1}{2}\left(1+\cos \phi_{x}\right)|0\rangle|0\rangle+\frac{1}{2} \sin \phi_{x}(|0\rangle|1\rangle+|1\rangle|0\rangle) \\
& +\frac{1}{2}\left(1-\cos \phi_{x}\right)|1\rangle|1\rangle,
\end{aligned}
$$

where $\phi_{x}=2 \pi x / 3(x=0,1,2)$, as the code word states with equal probability, and decodes them by the squareroot measurement defined mathematically by

$$
\left|\Pi_{y y}\right\rangle \equiv\left(\sum_{x}\left|\Psi_{x x}\right\rangle\left\langle\Psi_{x x}\right|\right)^{-\frac{1}{2}}\left|\Psi_{y y}\right\rangle,
$$

then $I\left(X^{2}: Y^{2}\right)=1.3690$ bits of information can be retrieved in principle. This is larger than twice of $C_{1}$ (=0.6454). The SQCG is $I_{2} / 2-C_{1}=0.0391$ which is expected to be accessible in laboratory.

The measurement basis Eq. (21) is explicitly written as

$$
\begin{aligned}
& \left|\Pi_{00}\right\rangle=a\left|\Psi_{00}\right\rangle+b\left|\Psi_{11}\right\rangle+b\left|\Psi_{22}\right\rangle \\
& \left|\Pi_{11}\right\rangle=b\left|\Psi_{00}\right\rangle+a\left|\Psi_{11}\right\rangle+b\left|\Psi_{22}\right\rangle \\
& \left|\Pi_{22}\right\rangle=b\left|\Psi_{00}\right\rangle+b\left|\Psi_{11}\right\rangle+a\left|\Psi_{22}\right\rangle
\end{aligned}
$$

where

$$
\begin{aligned}
& a=\frac{4+\sqrt{2}}{3 \sqrt{3}}, \\
& b=-\frac{2-\sqrt{2}}{3 \sqrt{3}} .
\end{aligned}
$$

These bases are entangled states and the measurement described by the POVM $\left\{\left|\Pi_{y y}\right\rangle\left\langle\Pi_{y y}\right|\right\}$ is a typical example of quantum collective decoding. The ternay code word states $\left\{\left|\Psi_{x x}\right\rangle\right\}$ can be described by the real vectors in a three dimensional space spanned by $\{|0\rangle|0\rangle,|0\rangle|1\rangle+$ $|1\rangle|0\rangle,|1\rangle|1\rangle\}$, as seen from Eq. (20). This ternary set is called the lifted trine, and provides interesting insights into quantum measurement problems as discussed by Shor [31, 32]. The measurement basis $\left\{\left|\Pi_{y y}\right\rangle\right\}$ forms another orthonormal basis set in the three dimensional space. $\left\{\left|\Psi_{x x}\right\rangle\right\}$ and $\left\{\left|\Pi_{y y}\right\rangle\right\}$ are geometrically depicted in Fig. 1(c). The code words $\left\{\left|\Psi_{x x}\right\rangle\right\}$ are distinguished by the projection to each of the nearest $\left|\Pi_{y y}\right\rangle$. The channel matrix $[P(y \mid x)]=\left|\left\langle\Pi_{y y} \mid \Psi_{x x}\right\rangle\right|^{2}$ is expressed as

$$
[P(y \mid x)]=\left[\begin{array}{ccc}
\cos ^{2} \frac{\gamma}{2} & \frac{1}{2} \sin ^{2} \frac{\gamma}{2} & \frac{1}{2} \sin ^{2} \frac{\gamma}{2} \\
\frac{1}{2} \sin ^{2} \frac{\gamma}{2} & \cos ^{2} \frac{\gamma}{2} & \frac{1}{2} \sin ^{2} \frac{\gamma}{2} \\
\frac{1}{2} \sin ^{2} \frac{\gamma}{2} & \frac{1}{2} \sin ^{2} \frac{\gamma}{2} & \cos ^{2} \frac{\gamma}{2}
\end{array}\right],
$$

where

$$
\begin{aligned}
& \cos \frac{\gamma}{2}=\frac{\sqrt{2}+1}{\sqrt{6}} \\
& \sin \frac{\gamma}{2}=\frac{\sqrt{2}-1}{\sqrt{6}} .
\end{aligned}
$$


In this noiseless model the channel is essentially a measurement channel whose ambiguity is due to nonorthogonality of the code word states.

While the square root measurement is simply expressed by the von Neumann measurement in the three dimensional real space in terms of Eqs. (22), this mathematical expression informs us of nothing special about physical implementations of the decoder. There may be many possible ways to realize effectively the measurement channel matrix of Eq. (24). One of systematic and straightforward ways is to express the original measurement bases as simple separable bases plus additional unitary transformation, and to convert the unitary transformation into a quantum circuit [7, 24]. Along this line, we derive a quantum circuit realizing $\left\{\left|\Pi_{y y}\right\rangle\right\}$.

Let us rewrite the POVM $\left\{\left|\Pi_{y y}\right\rangle\left\langle\Pi_{y y}\right|\right\}$ as

$$
\begin{aligned}
\left|\Pi_{00}\right\rangle & =\hat{U}^{\dagger}|0\rangle|0\rangle, \\
\left|\Pi_{11}\right\rangle & =\hat{U}^{\dagger}|0\rangle|1\rangle, \\
|S\rangle & =\hat{U}^{\dagger}|1\rangle|0\rangle, \\
\left|\Pi_{22}\right\rangle & =\hat{U}^{\dagger}|1\rangle|1\rangle,
\end{aligned}
$$

where $|S\rangle=(|0\rangle|1\rangle-|1\rangle|0\rangle) / \sqrt{2}$. The unitary operator $\hat{U}$ can be given by the matrix representation

$$
\hat{U}=\left[\begin{array}{cccc}
\cos \frac{\gamma}{2} & 0 & 0 & \sin \frac{\gamma}{2} \\
-\frac{1}{\sqrt{2}} \sin \frac{\gamma}{2} & \frac{1}{2} & \frac{1}{2} & -\frac{1}{\sqrt{2}} \cos \frac{\gamma}{2} \\
0 & \frac{1}{\sqrt{2}} & -\frac{1}{\sqrt{2}} & 0 \\
-\frac{1}{\sqrt{2}} \sin \frac{\gamma}{2} & -\frac{1}{2} & -\frac{1}{2} & -\frac{1}{\sqrt{2}} \cos \frac{\gamma}{2}
\end{array}\right]
$$

with respect to the separable basis $\{|0\rangle|0\rangle,|0\rangle|1\rangle,|1\rangle|0\rangle,|1\rangle|1\rangle\}$. Circuit construction for this unitary operator can be carried out in the following way. With the help of the Gaussian elimination algorithm [33], $\hat{U}$ can be decomposed into a product of $\mathrm{U}(2)$ operators $\hat{T}_{[j, i]}$ as

$$
\hat{U}=\hat{T}_{[2,1]} \hat{T}_{[3,1]} \cdots \hat{T}_{[4,2]} \hat{T}_{[4,3]}
$$

where $\hat{T}_{[j, i]}$ represents the two-dimensional rotation operators between the $i$-th and $j$-th basis vectors. Each operator $\hat{T}_{[j, i]}$ is then converted into a quantum circuit by using the formulae established by Barenco et al. [34]. The quantum circuit derived along this line consists of so many 2 bit basic gates, and is generally not in the minimal form. We further compiled the circuit into a much simpler version in a heuristic way. The final and possibly the simplest quantum circuit for $\hat{U}$ is shown in Fig. 2] It consists of five controlled-unitary gates.

\section{IMPLEMENTATION}

A favorable qubit trine is made of a flying qubit of photons. It would be natural to construct code words by the pairs of photons in the same linear polarization

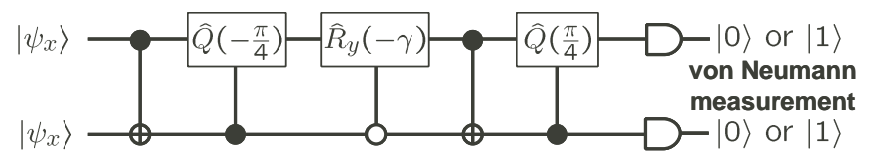

FIG. 2: Quantum circuit to realize the quantum collective decoding by the square root measurement $\left\{\left|\Pi_{y y}\right\rangle\right\}$. A received code word state is first transformed by the five controlled gates, and then is detected by a standard von Neumann measurement on each letter separately. Nomenclature of the controlled gates is based on Ref. [34]. $\hat{Q}(\varphi)$ is the unitary operator defined as $\hat{Q}(\varphi)=\hat{R}_{y}(\varphi) \hat{\sigma}_{z}$. The open circle notation indicates conditioning on the 'control' qubit being set to zero.

states. The quantum circuit of Fig. 2 then requires photon-photon gates. Although the principle of such photon-photon gates have been demonstrated experimentally [35], its precision is still far below the level required to access the small superadditive coding gain. Even if we rely on other, not flying, qubit systems such as trapped ion or molecules in NMR for which quantum gating has been demonstrated to date, it seems still formidable to run a five-step gating operation with the required precision.

Therefore we consider the length two coding based on the two physically different kinds of qubit, namely, a polarization and location qubits of a single photon. The first and second letter states of a code word are drawn from the ternary letter state sets of a polarization and a location qubits, $\left\{\left|\psi_{x}\right\rangle_{P}\right\}$ and $\left\{\left|\psi_{x}\right\rangle_{L}\right\}$, respectively. Then the collective decoding can be realized by an optical circuit consisting only of linear passive components, and a sufficiently high gating precision can be attained. In fact, by using the same polarization-location encoding format [36, 37, 38], several quantum algorithms have been demonstrated experimentally [39, 40].

In the following subsections, we first describe the physical implementation based on the polarization-location format. Then we discuss three kinds of experiments on quantum measurement of the accessible information, the single-shot capacity, and SQCG attained by the second order mutual information. These schemes are on a structured scenario of the capacity theory as described in Sec. III. They also correspond to the most typical measurements for the same qubit trine in the framework of quantum measurement theory, i.e. the von Neumann measurement, the single-shot generalized measurement, and the collective measurement.

\section{A. Preparation of optical qubit states}

The polarization qubit consists of the horizontal $|0\rangle_{P}=$ $|H\rangle$ and the vertical $|1\rangle_{P}=|V\rangle$ polarization states of a single photon and prepared by a half waveplate (HWP) 
which acts as

$$
\begin{aligned}
|H\rangle & \mapsto-\cos 2 \theta|H\rangle+\sin 2 \theta|V\rangle, \\
|V\rangle & \mapsto \sin 2 \theta|H\rangle+\cos 2 \theta|V\rangle,
\end{aligned}
$$

where $\theta$ is the angle of the fast axis from the vertical axis. The elements of the ternary set of polarization qubit $\left|\psi_{0}\right\rangle_{P},\left|\psi_{1}\right\rangle_{P},\left|\psi_{2}\right\rangle_{P}$ can be prepared from the input of the $|0\rangle_{P}$ state by setting $\theta=0, \pi / 6, \pi / 3[\mathrm{rad}]$, respectively.

The ternary set of location qubit $\left\{\left|\psi_{x}\right\rangle_{L}\right\}$ can be prepared by guiding the polarization letter states into two optical paths through a polarizing beam splitter (PBS). It reflects the vertical polarization and transmits the horizontal polarization as

$$
\begin{aligned}
\left.|H\rangle_{A} \otimes \mid \text { vacuum }\right\rangle_{B} & \left.\mapsto|H\rangle_{A} \otimes \mid \text { vacuum }\right\rangle_{B}, \\
\left.|V\rangle_{A} \otimes \mid \text { vacuum }\right\rangle_{B} & \mapsto i \mid \text { vacuum }\rangle_{A} \otimes|V\rangle_{B},
\end{aligned}
$$

where $\mathrm{A}$ and $\mathrm{B}$ are the labels for the two different optical paths.

The length two coding can be realized in the Hilbert space spanned by the orthonormal bases [36, 37, 38],

$$
\begin{aligned}
|00\rangle & \left.=|0\rangle_{P} \otimes|0\rangle_{L}=|H\rangle_{A} \otimes \mid \text { vacuum }\right\rangle_{B}, \\
|01\rangle & \left.=|0\rangle_{P} \otimes|1\rangle_{L}=\mid \text { vacuum }\right\rangle_{A} \otimes|H\rangle_{B}, \\
|10\rangle & \left.=|1\rangle_{P} \otimes|0\rangle_{L}=|V\rangle_{A} \otimes \mid \text { vacuum }\right\rangle_{B}, \\
|11\rangle & \left.=|1\rangle_{P} \otimes|1\rangle_{L}=\mid \text { vacuum }\right\rangle_{A} \otimes|V\rangle_{B} .
\end{aligned}
$$

In this space the encodings can be performed by the optical circuit shown in Fig. 3 which consists of a PBS and three HWPs. With an input photon initially in the state $|00\rangle$, the output of this encoder is given by

$$
\begin{aligned}
|\Psi\rangle= & \cos 2 \theta_{0} \cos 2 \theta_{1}|00\rangle-\sin 2 \theta_{0} \sin 2 \theta_{2}|01\rangle \\
& -\cos 2 \theta_{0} \sin 2 \theta_{1}|10\rangle+\sin 2 \theta_{0} \cos 2 \theta_{2}|11\rangle,
\end{aligned}
$$

where $\theta_{0}, \theta_{1}$ and $\theta_{2}$ are the angles of the three HWPs. By controlling these angles appropriately, polarization qubit states $\left|\psi_{x}\right\rangle_{P} \otimes|0\rangle_{L}$, location qubit states $|0\rangle_{P} \otimes\left|\psi_{x}\right\rangle_{L}$, and the length two code word states $\left|\psi_{x}\right\rangle_{P} \otimes\left|\psi_{x}\right\rangle_{L}$ can be prepared.

Thus in our coding format, doubling the transmission resource is realized by doubling the spatial resource instead of by using two polarized photons. From the viewpoint of communication theory, this can be regarded as a kind of pulse position coding which is often used when the signal power available is severely limited.

\section{B. Accessible information}

The POVM for the accessible information $I_{\text {Acc }}$ generally consists of overcomplete nonorthogonal states, which is a typical example of generalized measurement. It is well known that such a POVM can be implemented by a von Neumann measurement in an extended Hilbert space, which is called the Naimark extension [28]. The

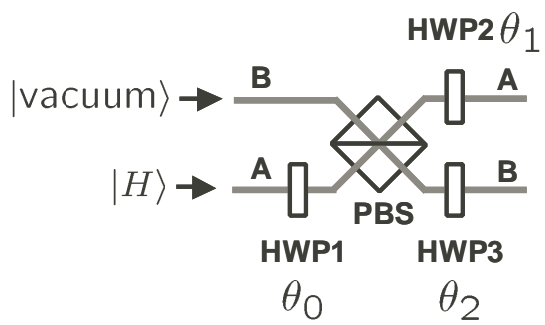

FIG. 3: Optical circuit for polarization-location encoding. HWP: half waveplate and PBS: polarizing beam splitter.

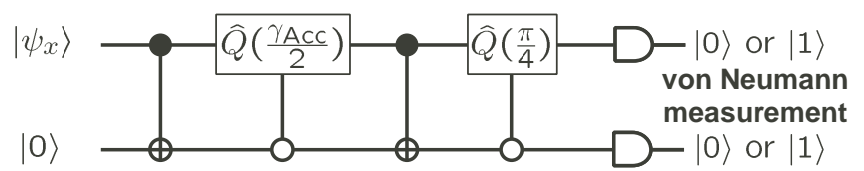

FIG. 4: Quantum circuit to realize the optimal POVM for the accessible information $\left\{\left|\omega_{y}\right\rangle\left\langle\omega_{y}\right|\right\}$ given in Eq. (17). Nomenclature of the gates is same as in Fig. 2]

experiments [29, 30] of such measurement were already performed by using polarization qubits. We have performed such experiment again for both polarization and location qubits. We also show the logical quantum circuit of such POVM.

The Naimark extension of the POVM $\left\{\left|\omega_{y}\right\rangle\left\langle\omega_{y}\right|\right\}$ given in Eq. (17) can be implemented by introducing an ancillary qubit with that in the initial state $|0\rangle$, and by constructing the orthonormal bases whose projection onto

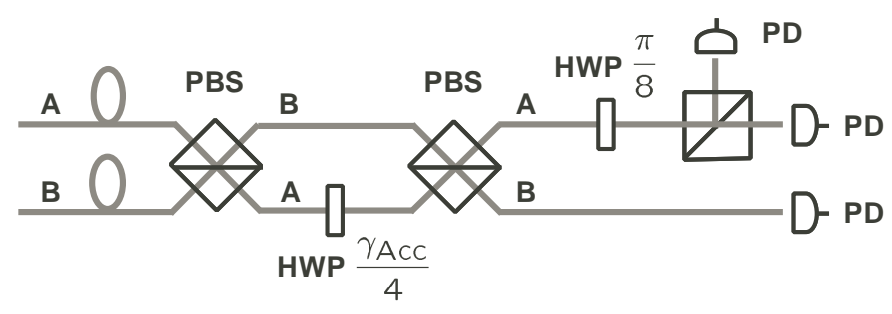

(a)

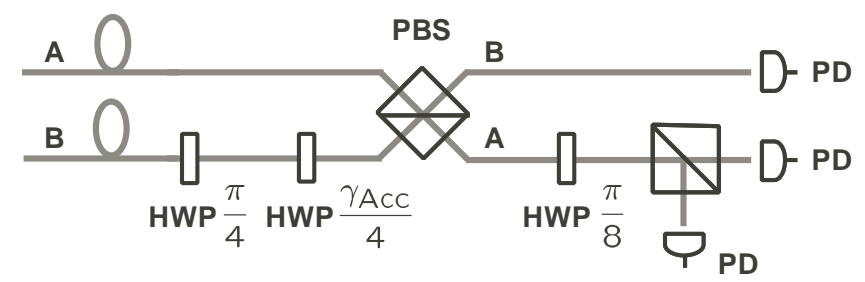

(b)

FIG. 5: Optical implementation of the circuit for the accessible information in Fig. 4 for (a) polarization qubit and (b) location qubit. PD: photodetector. 
the original plane becomes $\left\{\left|\omega_{y}\right\rangle\right\}$. The orthonormal bases are then decomposed into the unitary transformation and the von Neumann measurement by two qubit separable bases. The schematic of this process is shown in Fig. 4 where the unitary operation is described by the quantum circuit consisting of four controlled unitary gates. This one seems to be almost the simplest circuit. As discussed in the previous subsection, such quantum circuit can be translated into the optical circuits consisting of linear elements. Figures 5(a) and (b) show the optical circuits for polarization and location qubit signals, respectively.

The actual experimental setups for the polarization and location qubit trines are depicted in Fig. 6(a) and (b), respectively. In both setups, the left and right of the dashed vertical line correspond to the circuits for generating and measuring the signals, respectively. The signal states are generated by varying the angle of HWP1 with $\phi_{x}=2 \pi x / 3(x=0,1,2)$. It should be noted that, for practical reason, the original circuits in Fig. 5(a) and (b) are modified by using the 50:50 beam splitter (BS) instead of the PBS, and the initial state of the ancillary qubit is set to the state $|1\rangle$.

The experimental procedures and techniques are basically the same as those in Ref. 30]. The whole circuit in Fig. 6] consists of a polarization Mach-Zehnder interferometer and is controlled by a Piezoelectric transducer (PZT). The CW light from a He-Ne laser (SpectraPhysics, model 117A) operating at the wavelength of $632.8 \mathrm{~nm}$ with $1 \mathrm{~mW}$ power is strongly attenuated by ND filters with a factor of $5 \times 10^{-10}$ such that about $10^{-2}$ photons exist on average in the whole circuit. The attenuated light is purified to the horizontally polarized state by a Glan-Thompson prism and then injected to the interferometer. The HWP in the encoder (HWP1) is driven by a stepping motor to generate the signal state $\left\{\left|\psi_{x}\right\rangle_{P} \mid x=0,1,2\right\}$ or $\left\{\left|\psi_{x}\right\rangle_{L} \mid x=0,1,2\right\}$ sequentially. After passing through the circuit, the signal photons are guided into the silicon avalanche photodiodes (EG \& G, SPCM-AQ-141-FC), APD0-2, whose quantum efficiency and darkcount are typically $70 \%$ and 100 counts/sec, respectively, through a multimode optical fiber with coupling efficiency of about $80 \%$. The interferometer is enclosed in a darkened box. There are, however, background photons amount to about 300 counts/sec, even if no laser light is injected.

The mutual information is evaluated by constructing the $3 \times 3$ channel matrix $\left[P(y \mid x) \equiv\left|\left\langle\omega_{y} \mid \psi_{x}\right\rangle\right|^{2}\right]$ from a statistical data of single photon events detected by either of the three APDs conditioned on the input state $\left|\psi_{x}\right\rangle$. The mutual information thus obtained measures the ratio of number of bits retrieved per number of total photon counts. This event selection allows us to simulate communications of sending and detecting photons in pure state one by one through a noiseless channel even when a photon source with random arrival times 41. and photodetectors with imperfect efficiencies are used. The channel characteristics are then limited only by the

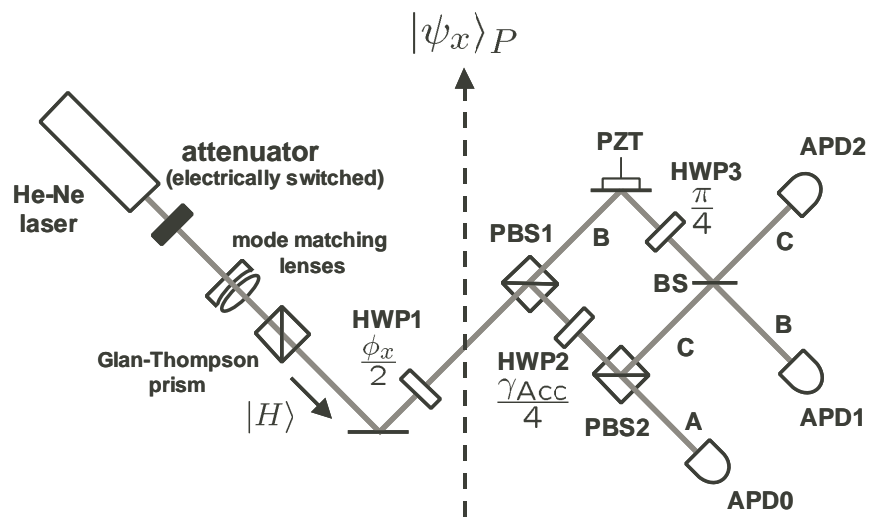

(a)

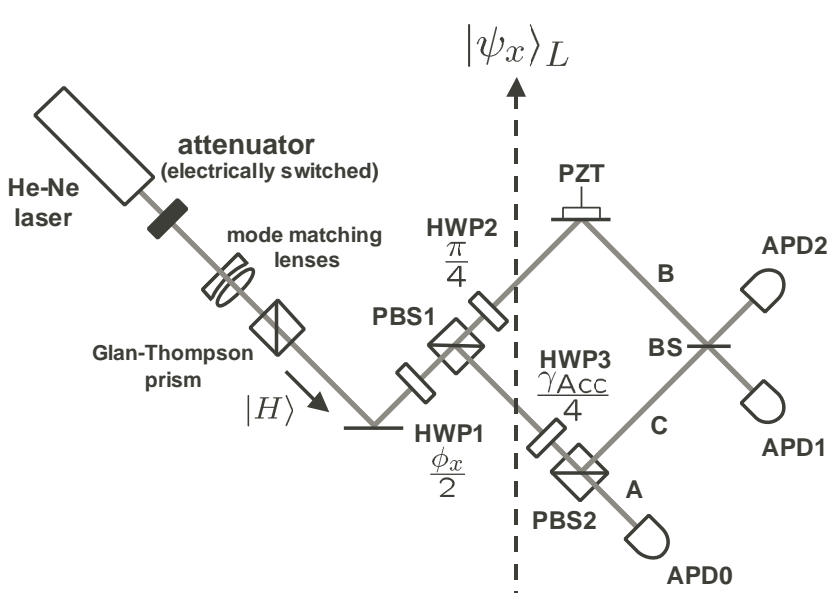

(b)

FIG. 6: Experimental setups for the accessible information for (a) polarization qubit and (b) location qubit. Here $\phi_{x}=$ $2 \pi x / 3+\phi_{\text {off }}(x=0,1,2)$ with the offset angle $\phi_{\text {off }}$. HWP: half waveplate, PBS: polarizing beam splitter, BS: 50:50 beam splitter, and PZT: Piezoelectric transducer.

non-commutativity of the signal states, imperfect alignment of the whole interferometer, deviation from the lock points, and the darkcount of the APDs.

The relative path length of the interferometer is adjusted to be a proper operating point by using a bright reference beam and the PZT. The visibility of the interferometer better than $98 \%$ is obtained. To circumvent injecting voltage noise from electronics, we simply used a low noise voltage source for adjusting and fixing mirror positions whereas an electrical feedback system was used in Ref. [30]. Once the circuit is adjusted, the reference beam is shut off. The signal light is then guided into the whole circuit. Photon counts are measured for five-second duration. This procedure is repeated for each letter state, composing a full sequence of measuring the channel matrix. The temporal stability corresponds to the change of the relative path length within $3 \mathrm{~nm}$ for at 
least more than $200 \mathrm{sec}$, which causes the error in mutual information \pm 0.005 bits at most.

Figures $\mathbf{7}(\mathrm{a})$ and (b) show the mutual informations measured for the polarization and location qubits, respectively. The offset angle of the horizontal axis is defined by the relative angle $\phi_{\text {off }}$ between the signal and measurement state sets (see Fig. [6] and its caption). The accessible informations is measured at the zero offset angles for both the polarization and the location qubits, and the results are $0.560 \pm 0.005 \mathrm{bits} /$ letter and $0.557 \pm 0.007$ bits/letter, respectively. The average visibilities at these points are evaluated to be $99.16 \%$ and $99.05 \%$, respectively. The difference between the data points and the ideal curve is mainly attributed to the imperfection of the PBSs. The result for the polarization qubit can directly be compared to the previous experiments [29, 30] and certifies that the quality of our interferometer is improved from those previous results.

\section{C. $C_{1}$ limit}

The classical limit of the capacity $C_{1} \quad$ (= $0.6454 \mathrm{bits} /$ letter) is obtained by sending only two of three letters with $1 / 2$ probabilities and a von Neumann measurement in the two-dimensional space (Fig. I(b)). The corresponding decoding circuits in the polarization and location qubits are shown in Figs. 8(a) and (b). For the polarization qubit (Fig. 8(a)), the polarization of the received photon is rotated by the HWP with $\pi / 12$ radians, and then is discriminated by the PBS followed by APDs. Detection of the location qubit is straightforward, i.e. just detecting a photon at each optical path. The measured value is $C_{1}=0.644 \pm 0.001 \mathrm{bits} /$ letter, for both the polarization and location qubits.

\section{Length two coding}

The length two code word states $\left|\Psi_{x x}\right\rangle \equiv\left|\psi_{x}\right\rangle_{P} \otimes\left|\psi_{x}\right\rangle_{L}$ are encoded by the optical circuit of Fig. [3 The angles of HWPs are set as

$$
\left\{\begin{array}{l}
\theta_{0}=\frac{1}{2} \arctan \sqrt{\frac{1-\cos \phi_{x}}{1+\cos \phi_{x}}} \\
\theta_{1}=\frac{1}{2} \arctan \left(\frac{-\sin \phi_{x}}{1+\cos \phi_{x}}\right) \\
\theta_{2}=\frac{1}{2} \arctan \left(\frac{-\sin \phi_{x}}{1-\cos \phi_{x}}\right)
\end{array}\right.
$$

where $\phi_{x}=2 \pi x / 3(x=0,1,2)$ corresponds to the encoding parameter in Eq. (20).

Figure 9 shows the optical circuit that corresponds to the collective decoding circuit in Fig. 2 It is further simplified for practical convenience, and the whole experimental setup including encoder is shown in Fig. 10] It includes a polarization and a normal interferometers in Mach-Zehnder arrangements. Each interferometer is aligned independently to achieve a visibility better than

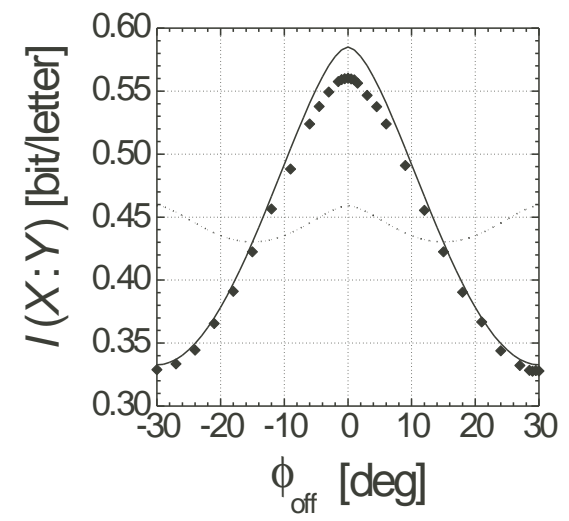

(a)

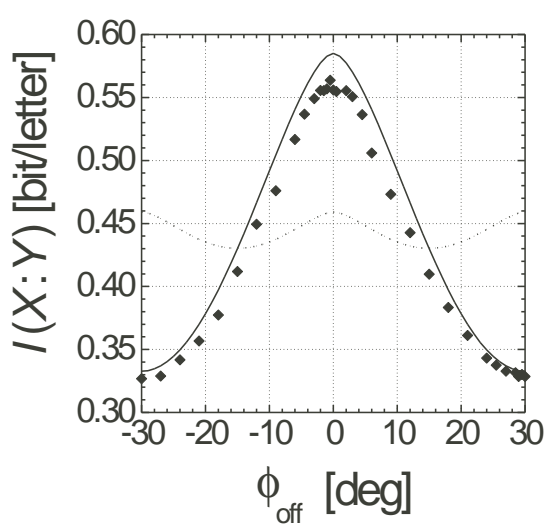

(b)

FIG. 7: Measured (filled diamonds) and theoretical (solid curve) accessible information for (a) the polarization qubit signal and (b) the location qubit signal as a function of the offset angle $\phi_{\text {off }}$. The dashed curve is the mutual information obtainable by a standard von Neumann measurement [30].

$98 \%$. Received code words are decided to be either of $\left|\Psi_{00}\right\rangle,\left|\Psi_{11}\right\rangle$, or $\left|\Psi_{22}\right\rangle$ according to the reception of the photon by APD0, APD1, APD2, respectively. Other procedures and conditions are the same as those of the experiment for the accessible information extraction.

Figure 11] shows a typical experimental data that corresponds to the unnormalized value of each element of the channel matrix $[P(y y \mid x x)]$ Ideally, the ratio of the diagonal and off-diagonal elements must be 0.9714 and 0.0143 , respectively. The total events counted for $1 \mathrm{sec}$ is of order $10^{6}$, while the average count for the off-diagonal elements is about $1.9 \times 10^{4}$ including the darkcounts of the three APDs. The total background count is $2 \%$ of the average count for the off-diagonal elements. The mutual information is evaluated as $I\left(X^{2}: Y^{2}\right)=1.312 \pm 0.005$ bits. The averaged visibility of the whole system is evaluated to be $98.48 \%$, which is slightly worse than the result for $I_{\text {Acc }}$. 


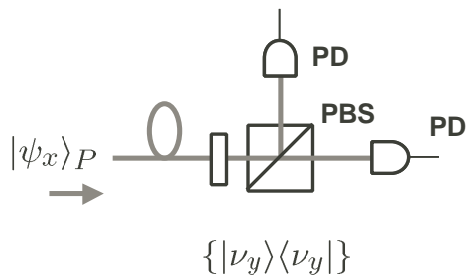

(a)

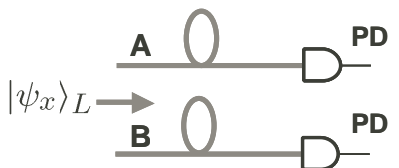

$\left\{\left|\nu_{y}\right\rangle\left\langle\nu_{y}\right|\right\}$
FIG. 8: Optical circuits for the $C_{1}$ detection. (a) Polarization qubit, (b) location qubit.

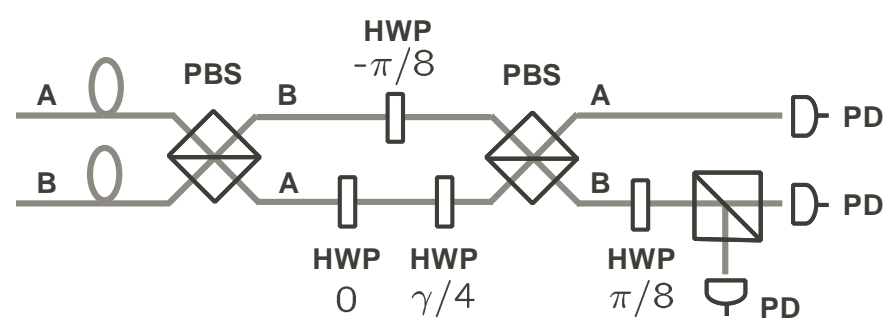

FIG. 9: Optical circuit for the collective decoding described by the square root measurement.

This degradation is mainly due to the relative difference of the polarization axis between two interferometers.

For experimental clarity, we measured the variation of the mutual information when the code word state set $\left\{\left|\Psi_{x x}\right\rangle\right\}$ is rotated relative to the decoder state set $\left\{\left|\Pi_{y y}\right\rangle\right\}$ around the vertical axis in Fig. 1(c). The rotation is achieved by adding an offset angle $\phi_{\text {off }}$ to $\phi_{x}$ in Eq. (33). Figure 12 shows the result in which the experimental data of the collective decoding (filled diamonds) are compared to its ideal curve (solid curve). The experimental and ideal values of $C_{1}$ and $I_{\text {Acc }}$ are also shown. The difference between the data points and the ideal curve is attributed again to the imperfection of the PBSs and also to the relative difference of the polarization axis between two interferometers. The obtained mutual information, $0.656 \pm 0.003 \mathrm{bits} /$ letter, exceeds the theoretical limit of the classical capacity $C_{1}=0.6454$ bits/letter. Classical length two coding corresponds to the use of polarization and location channels at a time in a separable decoding circuit construction, which does not include any entangling operation. Then the retrievable information can never exceed $2 C_{1}$. Our result, therefore, clearly shows the experimental evidence of the superadditivity, that is, the increase of information more than twice obtained by inserting an appropriate quantum circuit to entangle two letter states.

\section{QUANTUM-CLASSICAL HYBRID CODING}

The SQCG in small blocks is not only of proof-ofprinciple demonstration but also of practical importance in quantum-limited communications. Even two-qubit quantum circuit like Fig. 2 is useful in boosting the performance of a classical decoder. In this section, using the case of the ternary letter state set of Eq. (16), we show how the two-qubit quantum decoder can be combined with a classical decoder to improve the total communication performance.

Let us start with a classical channel coding of length $n$, whose schematic is shown in Fig. 13(a). We assume that the letter states are the ternary symmetric states given by linear polarizations of a single photon. A code word is then physically represented by a sequence of optical pulses of single photon, which is a tensor product of each letter state. At the receiving side, each optical pulse enters a photodiode, and is converted into an electric pulse, namely, a classical signal. This opto-electric conversion is made on each letter state separately. The classical electric signals are then processed by an electric circuit (a classical decoder) to reconstruct an output message.

The capacity attained by this classical coding scheme is given by the quantity $C_{1}$. As discussed in Sec. IV $C_{1}$ (=0.6454 bits/letter) is attained by making the code words from only two letter states instead of using all three, and by performing the binary measurement. In other words, the channel is equivalent to the binary symmetric channel whose error probability is characterized by

$$
\begin{aligned}
\epsilon & \equiv P(1 \mid 0)=P(0 \mid 1) \\
& =\frac{1}{2}\left(1-\sqrt{1-\left|\left\langle\psi_{0} \mid \psi_{1}\right\rangle\right|^{2}}\right)=\frac{2-\sqrt{3}}{4}
\end{aligned}
$$

The transmission rate for this channel is defined by $R^{\mathrm{C}}=$ $k / n$. So if $R^{\mathrm{C}}<C_{1}=0.6454$, one must be able to find codes for which the decoding error approaches zero in the limit $n \rightarrow \infty$.

In practice, however, one wants to know the decoding error for finite length $n$. According to the theory of reliability function 2], there exists a length $n$ (classical) coding attaining $P_{\mathrm{e}} \leq 2^{-n E_{r}(R)}$, where $E_{r}(R)$ is the lower bound of the reliability function. The function $E_{r}(R)$ is useful to investigate the maximum communication performance of channels for a given code length.

Let us denote the function $E_{r}(R)$ for the classical decoding scheme as $E_{r}^{\mathrm{C}}\left(R^{\mathrm{C}}\right)$. As for the definition of $E_{r}(R)$ and the expression of $E_{r}^{\mathrm{C}}\left(R^{\mathrm{C}}\right)$, see Appendix. We now consider, as examples, two cases where transmission rates are low $\left(R^{\mathrm{C}}=0.1=0.15 \times C_{1}\right)$ and high $\left(R^{\mathrm{C}}=0.62=0.96 \times C_{1}\right)$. In these cases, we obtain $E_{r}^{\mathrm{C}}(0.1)=5.218 \times 10^{-4}$ and $E_{r}^{\mathrm{C}}(0.62)=0.3150$, respectively, and the asymptotic behaviors of the decoding error probabilities are shown in Fig. 14 (the dashed curves). This is a typical error performance obtained by averaging over all possible classical codes. It means that there 


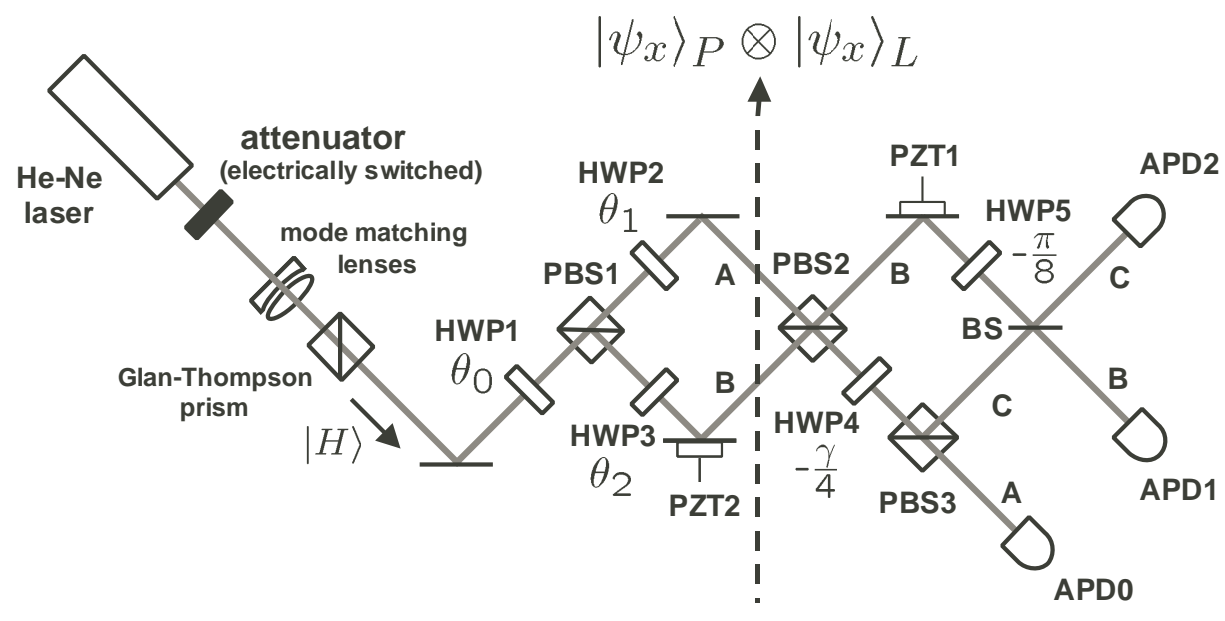

FIG. 10: Experimental setup. HWP: half waveplate, PBS: polarizing beam splitter, BS: 50:50 beam splitter, and PZT: Piezo transducer. The HWP angles $\theta_{0}-\theta_{2}$ are given in the text.

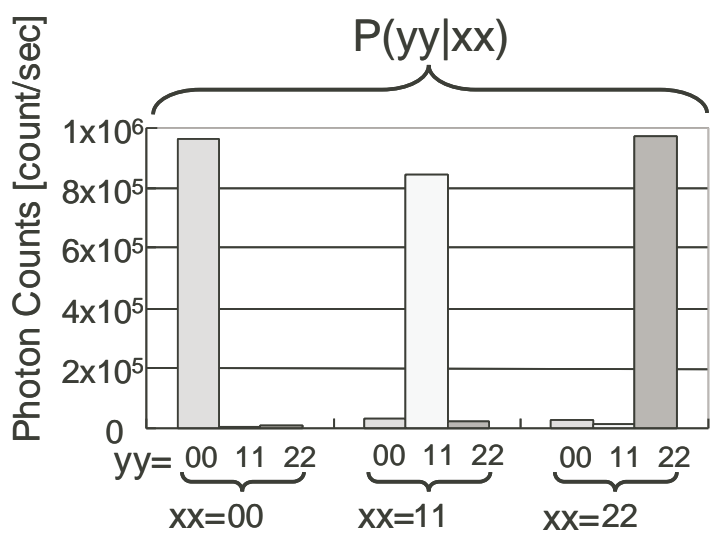

FIG. 11: Histogram of photon counts for the channel matrix elements $P(y y \mid x x)=\left|\left\langle\Pi_{y y} \mid \Psi_{x x}\right\rangle\right|^{2}$ (unnormalized).

must exist at least one code that exhibits a performance superior to that shown in Fig. 14]

Now we turn to the other scheme, in which the above classical coding is combined with quantum channel coding. We call such a combination of two coding schemes as quantum-classical hybrid coding (QCHC). In the following, we show the power of QCHC by discussing the QCHC scheme with the length two quantum coding. Its schematic is shown in Fig. 13 (b). Given the length $n$ (assumed to be an even number), we consider a classical coding of length $n / 2$ with the composite letters $\{00,11,22\}$. In decoding, the received code word state is first processed by the two-qubit quantum circuit shown in Fig. 2 and then is detected by two photodiodes. This is the quantum collective decoding consisting of the square root measurement. The resulting $n / 2$ electric pulses are finally processed by a classical decoder.

The channel model of this scheme is equivalent to a

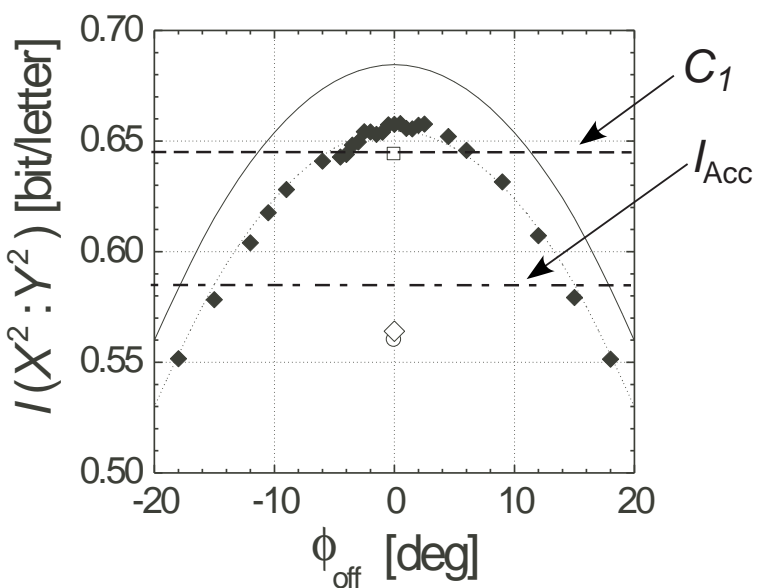

FIG. 12: Measured (filled diamonds) and theoretical (solid curve) mutual information as a function of the offset angle of the code word state set $\left\{\left|\Psi_{x x}\right\rangle\right\}$ from the decoder state set $\left\{\left|\Pi_{y y}\right\rangle\right\}$ around the vertical axis in Fig. 1(c). The dotted curve is just the guide for eyes. The experimental and theoretical $C_{1}$ are shown by the square and the dashed line, respectively. The accessible information $I_{\text {Acc }}$ experimentally observed for polarization and location qubit, and theoretically predicted are shown by the open diamond, open circle, and one-dotted line, respectively.

classical coding of length $n / 2$ based on the ternary symmetric channel given by Eq. (24). The transmission rate for this channel is now defined by $R^{\mathrm{QC}}=(k / n) \log _{2} 3$ and it can be raised up to the mutual information of this channel, $I\left(X^{2}: Y^{2}\right)=1.3690$. It means that the rate $k / n$ can be raised up to $I\left(X^{2}: Y^{2}\right) / \log _{2} 3=0.8637$.

Let us denote the function $E_{r}(R)$ for the QCHC as $E_{r}^{\mathrm{QC}}\left(R^{\mathrm{QC}}\right)$. The expression of $E_{r}^{\mathrm{QC}}\left(R^{\mathrm{QC}}\right)$ is also given 


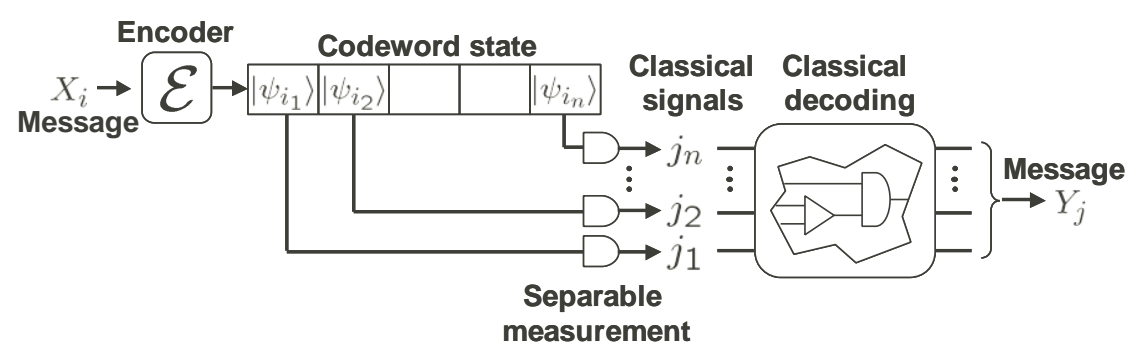

(a)

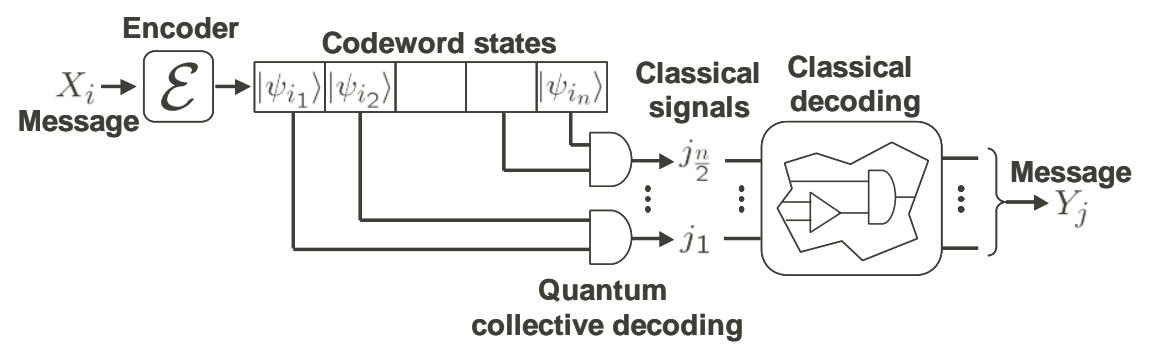

(b)

FIG. 13: (a) Classical channel coding system. (b) Quantum-classical hybrid channel coding system with the letter two quantum collective decoding.

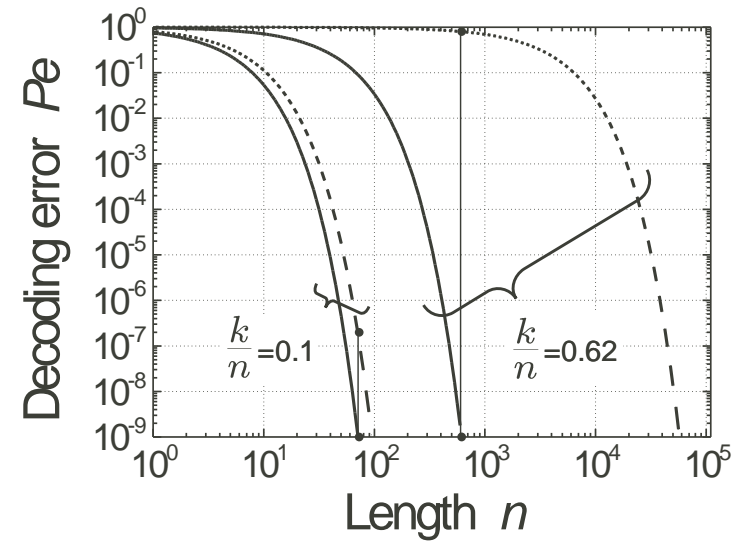

FIG. 14: Decoding error probabilities with finite code length for the QCHC (solid lines) and the all classical coding (dashed lines).

in Appendix. To compare the performance of $E_{r}^{\mathrm{QC}}\left(R^{\mathrm{QC}}\right)$ with that of $E_{r}^{\mathrm{C}}\left(R^{\mathrm{C}}\right)$, the rate $k / n$ should be fixed. For the same rates as before, $k / n=0.1$ and $k / n=0.62$, the transmission rates are given by $R^{\mathrm{QC}}=0.1585$ and $R^{\mathrm{QC}}=0.9827$, respectively, The function $E_{r}^{\mathrm{QC}}\left(R^{\mathrm{QC}}\right)$ at these rates are evaluated to be $E_{r}^{\mathrm{QC}}(0.1585)=0.8415$ and $E_{r}^{\mathrm{QC}}(0.9827)=9.753 \times 10^{-2}$, respectively. The upper bound of the error probability is then given by
$P_{\mathrm{e}}=2^{-(n / 2) E_{r}^{\mathrm{QC}}}$. The error probabilities for these examples are shown by the solid curves in Fig. 14]

As seen in this figure, at the rate of $k / n=0.62$, the decoding error starts to decrease rapidly over $n \sim 10000$ in the classical coding scheme. In the QCHC scheme, the error starts to decrease from $n \sim 100$, and reaches the standard error-free criterion $10^{-9}$ around $n \sim 600$ (300 composite letter pairs). With this code length, it is impossible to transmit any information reliably by the classical coding scheme 42. This improvement can be achieved just by inserting the two-qubit quantum decoder in front of the classical decoder. To achieve the standard error-free criterion by classical coding, one must use code words of length $n \sim 57300$.

As codes get longer, the complexity of the decoder, such as the total number of arithmetic operations $\chi(n)$, increases and eventually limits the effective transmission speed. The total decoding time per letter by the device with finite speed $\tau_{0}$ sec/step is given by $\frac{\chi(n)}{n} \tau_{0}$ sec/letter. Presumably, this is a limiting factor and thus we can define the effective transmission speed as $R_{\mathrm{eff}}=R \frac{n}{\chi(n) \tau_{0}}$ bits/sec. For some asymptotically good codes, the total number of arithmetic operations is typically of order $\chi(n)=O\left((n \log n)^{2}\right)$ [43]. Therefore, the effective transmission speed behaves as $R_{\text {eff }} \propto \frac{R}{n(\log n)^{2}}$. Then the reduction of code length brought by $\mathrm{QCHC}$ will be practically significant in the trade-off between performance and complexity. In our example, even in the lower rate, the decoding error of the $\mathrm{QCHC}$ around the standard error free criterion is two figures smaller than that of the 
classical coding with the same code length.

\section{CONCLUDING REMARKS}

In this paper, we have detailed the experimental demonstration of the superadditive quantum coding gain (SQCG). The superadditive quantum coding becomes essential in the region where the noncommutativity of signal states is the main ambiguity among signals. As a typical example, we have mentioned deep space optical communications in Sec. [I where we have to extract as much information as possible from the sequences of heavily attenuated signals. Apparently, it is not realistic in such a situation to transmit nonclassical states or to install quantum repeaters. Therefore, only quantum decoding can be the core technology to achieve the ultimate performance in future long-haul optical communications.

Quantum-classical hybrid coding (QCHC) is then a promising approach. We have shown in Section VI that even a small scale quantum computing can enhance the effective transmission speed when it is used together with large scale classical coding. Thus the QCHC allows one to extend conventional optical communications technology to the quantum limit in a straightforward way. This may be contrasted to the fact that other known quantum algorithms exhibit the advantage over their classical counterparts only when a large scale quantum computer is available. For the QCHC applications, the communication performance of QCHC gets better as available scale of quantum computer gets larger.

Finally, we mention the challenges to be overcome to bring a QCHC system into reality. Although our result clearly demonstrated the principle of SQCG, the physical scheme used in our experiment is still not suitable for real applications, i.e. is not applicable to weak coherent signals. To implement a quantum collective decoder for weak coherent signals, one must be able to entangle weak coherent optical pulses with respect to the degrees of freedom of phase and/or amplitude. Heavily attenuated coherent signals can be approximated by superpositions of zero and one photon states as $\left|\alpha_{k}\right\rangle \approx|0\rangle+\alpha_{k}|1\rangle$. It means that the proposals of qubit gating may be applicable to our problem.

Quantum gating operation at single photon level has been investigated by many authors, but it still remains as a challenging topic. One scenario suggested in Ref. 8] is to transfer the information in optical field to a multilevel single atom inside a high-finesse optical cavity in order to perform gating operations by Raman process. Another possible way is to use atomic system as a nonlinear medium with the idea of electromagnetically induced transparency [4]. Finally, a recent proposal suggests the possibility of an optical quantum circuit based on linear optics [45]. As single photon on-demand sources [46] and highly efficient photon detectors become available, small scale quantum gating circuits for coherent state signals can be realized, in principle, with only linear optics.
From the viewpoint of coding theory, on the other hand, it is still open to find asymptotically good quantum channel codes. In the case of pure state channels, Ref. [15] tells us that the problem is essentially the selection of appropriate sequences for code words, and the square root measurement does the decoding. It is also important to establish a systematic theory to synthesize a quantum circuit for intermediate scale collective decoding.

\section{APPENDIX: LOWER BOUND OF THE RELIABILITY FUNCTION}

In this Appendix, we give the definition of reliability function and its lower bound, $E_{r}(R)$. The latter one is applied to the channels describing the classical coding and the QCHC with the length two quantum coding, respectively, for the qubit trine signal.

The reliability function is defined as [2],

$$
E(R)=\lim _{n \rightarrow \infty} \sup \frac{-\ln P_{e}(n, R)}{n},
$$

where $P_{e}(n, R)$ is the minimum error probability over all $(n, R)$ codes. Although the true $E(R)$ for any $R$ has not been clarified yet, it is known that its lower bound is given by

$$
E_{r}(R)=\max _{\rho} \max _{\{P(x)\}}\left[E_{0}(\rho)-\rho R\right],
$$

where

$$
E_{0}(\rho, P(x))=-\log \sum_{y}\left(\sum_{x} P(x) P(y \mid x)^{1 /(1+\rho)}\right)^{(1+\rho)},
$$

with $0<\rho \leq 1$. The function $E_{r}(R)$ yields the upper bound of an average error probability, $P_{\mathrm{e}}$, for the code with given $n$ and $R$ by $P_{\mathrm{e}} \leq 2^{-n E_{r}(R)}$. We also note that, for any symmetric channels, $E_{r}(R)$ is maximized when the all signals are given by the equal prior probability distribution [2].

As discussed in Sec. [V] the channel matrix attaining the capacity of the classical coding for the qubit trine signal is given by the binary symmetric channel with the channel matrix

$$
[P(y \mid x)]=\left[\begin{array}{cc}
1-\epsilon & \epsilon \\
\epsilon & 1-\epsilon
\end{array}\right],
$$

where $\epsilon$ is given in Eq. (34). The analytic expression of $E_{r}(R)$ for this channel can easily be derived. First we define the quantities,

$$
\epsilon_{\rho} \equiv \frac{\epsilon^{1 /(1+\rho)}}{\epsilon^{1 /(1+\rho)}+(1-\epsilon)^{1 /(1+\rho)}},
$$

and

$$
R_{0} \equiv 1-H\left(\epsilon_{1}\right),
$$


where

$$
H\left(\epsilon_{\rho}\right) \equiv-\epsilon_{\rho} \log _{2} \epsilon_{\rho}-\left(1-\epsilon_{\rho}\right) \log _{2}\left(1-\epsilon_{\rho}\right) .
$$

After maximizing $E_{0}(\rho, P(x))$ over $P(x)$ and $\rho$, we obtain that if $R<R_{0}$,

$$
E_{r}^{\mathrm{C}}(R)=1-2 \log _{2}(\sqrt{\epsilon}+\sqrt{1-\epsilon})-R,
$$

and if $R_{0}<R<C_{1}$,

$$
E_{r}^{\mathrm{C}}(R)=\epsilon_{\rho^{*}} \log _{2} \frac{\epsilon_{\rho^{*}}}{\epsilon}+\left(1-\epsilon_{\rho^{*}}\right) \log _{2} \frac{1-\epsilon_{\rho^{*}}}{1-\epsilon},
$$

where $\rho^{*}$ is the solution of

$$
R=1-H\left(\epsilon_{\rho}\right) .
$$

The channel matrix for the QCHC discussed in Sec. VI is given in Eq. (24), which is a ternary symmetric channel. The expression of $E_{r}(R)$ for a ternary symmetric channel can also be derived with the quantities,

$$
\Gamma_{\rho}=\frac{\left(\sin ^{2} \frac{\gamma}{2}\right)^{1 /(1+\rho)}}{\frac{1}{2}\left(2 \cos ^{2} \frac{\gamma}{2}\right)^{1 /(1+\rho)}+\left(\sin ^{2} \frac{\gamma}{2}\right)^{1 /(1+\rho)}},
$$

and

$$
R_{0} \equiv \log _{2} 3-\Gamma_{1}-H\left(\Gamma_{1}\right)
$$

Then if $R<R_{0}$,

$$
E_{r}^{\mathrm{QC}}(R)=\log _{2} 3-2 \log _{2}\left(\cos \frac{\gamma}{2}+\sqrt{2} \sin \frac{\gamma}{2}\right)
$$

and if $R_{0}<R<I\left(X^{2}: Y^{2}\right)$,

$$
E_{r}^{\mathrm{QC}}(R)=\Gamma_{\rho^{*}} \log _{2} \frac{\Gamma_{\rho^{*}}}{\sin ^{2} \frac{\gamma}{2}}+\left(1-\Gamma_{\rho^{*}}\right) \log _{2} \frac{1-\Gamma_{\rho^{*}}}{\cos ^{2} \frac{\gamma}{2}}
$$

where $\rho^{*}$ is the solution of

$$
R=\log _{2} 3-\Gamma_{\rho}-H\left(\Gamma_{\rho}\right)
$$

[1] C. E. Shannon, Bell System Tech. J. 27, 379 (Part I) and 623 (Part II) (1948).

[2] R. G. Gallager: Information Theory and Reliable Communication (John Wiley and Sons, New York, 1968).

[3] T. Cover and J. Thomas: Elements of Information Theory (John Wiley and Sons, New York, 1991).

[4] A. S. Holevo, Probl. Peredachi Inform. 15(4), 3 (1979). (English transl.: Problems of Inform. Transm., 15(4), 247 (1980).)

[5] A. Peres and W. K. Wootters, Phys. Rev. Lett. 66, 1119 (1991).

[6] M. Sasaki, K. Kato, M. Izutsu, and O. Hirota, Phys. Lett. A 236, 1 (1997).

[7] M. Sasaki, K. Kato, M. Izutsu, and O. Hirota, Phys. Rev. A 58, 146 (1998).

[8] J. R. Buck, S. J. van Enk, and C. A. Fuchs, Phys. Rev. A 61, 032309 (2000).

[9] S. Usami, T. S. Usuda, I. Takumi, R. Nakano, and M. Hata, Quantum Communication, Computing, and Measurement 3 (Eds. Tombesi, P., and Hirota, O. Kluwer academic/Prenum, New York, 2001) 35; T. S. Usuda, S. Usami, I. Takumi, and M. Hata, Phys. Lett. A 305, 125 (2002).

[10] C. W. Helstrom, Quantum Detection and Estimation Theory (Academic Press, New York, 1976).

[11] J. P. Gordon, IRE Proc. 50, 1898 (1962).

[12] J. P. Gordon, Quantum Electronics and Coherent Light, Proc. Int. School Phys. "Enrico Fermi", Course XXXI, (Ed. P. A. Miles, New York: Academic Press 1964) 156.

[13] D. S. Lebdev and L. B. Levitin, Inform. Contr. 9, 1 (1966).

[14] A. S. Holevo, Probl. Peredachi Inform. 9(3), 3 (1973). (English transl.: Problems of Inform. Transm., 9(3), 177 (1973).)
[15] P. Hausladen, R. Jozsa, B. Schumacher, M. Westmoreland, and W. K. Wootters, Phys. Rev. A 54, 1869 (1996).

[16] B. Schumacher and M. D. Westmoreland, Phys. Rev. A 56, 131 (1997).

[17] A. S. Holevo, IEEE Trans. Inf. Theory IT-44, 269 (1998).

[18] M. Fujiwara, M. Takeoka, J. Mizuno, and M. Sasaki, Phys. Rev. Lett. 90, 167906 (2003).

[19] H. Barnum, M. A. Nielsen, and B. Schumacher, Phys. Rev. A 57, 4153 (1998).

[20] C. H. Bennett and S. J. Wiesner, Phys. Rev. Lett.69, 2881 (1992).

[21] C. H. Bennett, G. Brassard, C. Crepeau, R. Jozsa, A. Peres, and W. K. Wootters, Phys. Rev. Lett. 70, 1895 (1993).

[22] C. H. Bennett, P. W. Shor, J. A. Smolin, and A. V. Thapliyal, IEEE Trans. Inf. Theory IT-48, 2637 (2002).

[23] A. Fujiwara and H. Nagaoka, IEEE Trans. Inf. Theory IT-44, 1071 (1998).

[24] M. Sasaki, T. Sasaki-Usuda, M. Izutsu, and O. Hirota, Phys. Rev. A 58, 159 (1998).

[25] E. B. Davies, IEEE Trans. Inf. Theory IT-24, 596 (1978).

[26] L. B. Levitin, Quantum Communication, and Measurement (Eds. V. P. Belavkin, O. Hirota, and R. L. Hudson, Prenum, New York, 1995), 439.

[27] M. Osaki, M. Ban, and O. Hirota, Quantum Communication, Computing, and Measurement 2 (Eds. P. Kumar, G. M. D'Ariano, and O. Hirota, Kluwer academic/Prenum publishers, New York, 2000) 17.

[28] M. Sasaki, S.M. Barnett, R. Jozsa, M. Osaki, and O. Hirota, Phys. Rev. A 59, 3325 (1999).

[29] R. B. M. Clarke, V. M. Kendon, A. Chefles, S. M. Barnett, E. Riis, and M. Sasaki, Phys. Rev. A 64, 012303 (2001).

[30] J. Mizuno, M. Fujiwara, M. Akiba, T. Kawanishi, 
S. M. Barnett, and M. Sasaki, Phys. Rev. A 65, 012315 (2001).

[31] P. W. Shor, LANL arXiv:quant-ph/0206058

[32] P. W. Shor, Quantum Communication, Computing, and Measurement 3 (Eds. O. Hirota and P. Tombesi, Kluwer, Dordrecht, 2001) 107. Also available at LANL arXiv:quant-ph/0009077

[33] M. Reck, A. Zeilinger, H. J. Bernstein, and P. Bertani, Phys. Rev. Lett. 73, 58 (1994).

[34] A. Barenco, C. H. Bennett, R. Cleve, D. P. DiVincenzo, N. Margolus, P. Shor, T. Sleator, J. A. Smolin, and H. Weinfurter, Phys. Rev. A 52, 3457 (1995).

[35] Q. A. Turchette, C. J. Hood, W. Lange, H. Mabuchi, and H. J. Kimble, Phys. Rev. Lett. 75, 4710 (1995).

[36] S. Takeuchi, Proceedings of the Fourth Workshop on Physics and Computation: PhysComp96, (Ed. T. Toffoli, New England Complex Systems Institute, Boston, 1996), 299.

[37] N. J. Cerf, C. Adami, and P. G. Kwiat, Phys. Rev. A 57, R1477 (1998).

[38] R. J. C. Spreeuw, Found. Phys. 28, 361 (1998);

[39] S. Takeuchi, Phys. Rev. A 62, 032301 (2000).

[40] P. G. Kwiat, J. R. Mitchell, P. D. D. Schwindt, and A. G. White, J. Mod. Opt. 47, 257 (2000).

[41] We note that the state of a temporal mode at the output of a laser operating far above its threshold is described as a Poisson-distributed, mixed photon number state. This description is further reinforced in our experiment by the use of photodetectors which project (destructively) the state of the temporal modes onto number states. Also, nowhere in our experiment do we determine the phase of the laser, hence a description of the output in terms of, say, coherent states is inappropriate. Thus, the light source in our experiment closely approximates a single photon source, albeit with the photons arriving at random times.

[42] Rigorously speaking, the optimal measurement for $C_{1}$ may not give the maximum $E$ for any transmission rate. Nevertheless, it gives almost maximum $E$, especially, in the region of high transmission rate since the capacity is the maximum transmission rate giving a non-negative $E$. Therefore, the discussions on the advantage of the $\mathrm{QCHC}$ is valid. To find the optimal measurement which maximizes $E$ is still an open problem.

[43] S. Hirasawa, M. Kasahara, Y. Sugiyama, and T. Namekawa, IEEE Trans. Inf. Theory IT-26, 527 (1980).

[44] M. Paternostro, M. S. Kim, and B. S. Ham, Phys. Rev. A 67, 023811 (2003).

[45] E. Knill, R. Laflamme, and G. J. Milburn, Nature 409, 46 (2001).

[46] J. Kim, O. Benson, H. Kan, and Y. Yamamoto, Nature 397, 500 (1999). 